This is an electronic reprint of the original article. This reprint may differ from the original in pagination and typographic detail.

Author(s): Portegijs, Erja; Rantanen, Taina; Sipilä, Sarianna; Laukkanen, Piia; Heikkinen, Eino

Title: $\quad$ Physical activity compensates for increased mortality risk among oder people with poor muscle strength

Year: $\quad 2007$

Version:

Please cite the original version:

Portegijs, E., Rantanen, T., Sipilä, S., Laukkanen, P., \& Heikkinen, E. (2007). Physical activity compensates for increased mortality risk among oder people with poor muscle strength. Scandinavian Journal of Medicine \& Science in Sports, 17, 473-479.

All material supplied via JYX is protected by copyright and other intellectual property rights, and duplication or sale of all or part of any of the repository collections is not permitted, except that material may be duplicated by you for your research use or educational purposes in electronic or print form. You must obtain permission for any other use. Electronic or print copies may not be offered, whether for sale or otherwise to anyone who is not an authorised user. 
Scandinavian Journal of Medicine and Science in Sports 2007;17:473-479.

DOI: $10.1111 / j .1600-0838.2006 .00606 . x$

\section{Physical activity compensates for increased mortality risk among older people with poor muscle strength}

Erja Portegijs *\#, Taina Rantanen ${ }^{* \#}$, Sarianna Sipilä ${ }^{* \#}$, Pia Laukkanen ${ }^{\S}$, Eino Heikkinen ${ }^{*}$

* Department of Health Sciences, University of Jyväskylä, Jyväskylä Finland

\# Finnish Center for Interdisciplinary Gerontology, University of Jyväskylä, Jyväskylä, Finland $\S$ Health Center of the City of Jyvaskyla, Jyvaskyla, Finland

Short running head-line: physical activity, strength and mortality

Corresponding author: Erja Portegijs

Department of Health Sciences

Finnish Center for Interdisciplinary Gerontology

University of Jyväskylä

PO. 35 (viv), FI-40014 Jyväskylä Finland

Phone: + 35814260 2175, Fax: + 358142604600

E-mail: erja.portegijs@sport.jyu.fi 


\begin{abstract}
The aim of the study was to determine whether habitual physical activity can compensate for the increased mortality risk among older people with poor muscle strength. Mortality was followedup for 10 years after laboratory examination in 558 community-dwelling 75- and 80-year-old men and women. Maximal isometric strength of 5 muscle groups was measured and tertile cutoff points were used to categorize participants. Participants, who reported moderate physical activity for at least 4 hours a week, were categorized as physically active and the others as sedentary. High muscle strength and physical activity both protected from mortality, but their effect was not additive. Within each muscle strength tertile, physically active people had a lower mortality risk than sedentary people, the effect being most pronounced among those with lower strength in all muscle groups. A high level of physical activity may thus compensate for the increased mortality associated with low muscle strength.
\end{abstract}

Keywords: physical exercise, sedentary, muscle force, survival, aged 


\section{Introduction}

With increasing age physical function declines and a sedentary lifestyle becomes more prevalent (DiPietro 2001). Physical activity may help to counteract the age-related decline in or improve muscle, cardio-vascular, respiratory and metabolic function. Additionally, physical activity may inhibit the onset of certain diseases as well as improve health and function among people with diseases, disabilities or depressive symptoms (e.g. reviewed by the American College of Sports Medicine, 1998; Bean et al., 2004; Taylor et al., 2004). Consequently, longitudinal studies have demonstrated that high physical activity is protective for all-cause mortality (e.g. reviewed by Lee \& Skerrett, 2001) and mortality from major chronic diseases such as cardiovascular disease (Kaplan et al., 1996; Kushi et al., 1997; Bijnen et al., 1998; Greg et al., 2003), respiratory disease (Kushi et al., 1997), and cancer (Greg et al., 2003).

Though sedentary lifestyle and poor muscle strength often coexist, they do not correlate fully (Rantanen et al. 1997). Some people with good muscle strength may be quite sedentary, while some people with low strength are physically quite active. Multiple factors underlying the individual differences in strength, including genetic factors (Tiainen et al., 2004), are a potential explanation for this. Previous longitudinal analyses have shown that the association of strength and all-cause mortality persisted even after adjustment for multiple factors potentially explaining the association, such as age, presence of chronic conditions, inflammation, nutritional status and depressive symptoms (e.g. Rantanen et al., 2003). Therefore it is possible that strength may be a constitutional marker of vulnerability to adverse health events, such as disease, disability, falls and injury, or poor recovery from these conditions, thus also increasing mortality risk. 
The aim of the study was to examine the combined effects of physical activity and muscle strength on all-cause mortality. The analyses were carried out to better understand whether physical activity may modify the risk of mortality among people with different levels of strength. 


\section{Methods}

\section{Participants}

This study is part of the Evergreen project, a longitudinal study on health and functional capacity among older residents in the city of Jyväskylä, Finland. A detailed description of the study design has been reported elsewhere (Heikkinen, 1997). Briefly, all community-dwelling people of the city aged 75 and 80 years were invited to participate in the study and 663 people were found eligible. In the autumn of 1989 and the winter of 1990, 93\% (n=617) of the eligible population agreed to participate and were interviewed at home. Ninety percent of the participants responded to the question about physical activity (men: 109 aged 75 years and 67 aged 80 years; and women: 204 aged 75 years and 178 aged 80 years $)$ and $81 \%(n=500)$ of them visited the laboratory for examination. Participation in the muscle strength measurements was contraindicated for 6 women and 9 men due to cardiovascular diseases, musculoskeletal problems or poor cooperation (Rantanen et al., 1994, Rantanen et al., 1998). Handgrip strength was the muscle strength test most commonly taken and was measured in 101 men aged 75 years and 55 aged 80 years, and in 186 women aged 75 years and 136 aged 80 years. In total, 478 people participated in at least 1 strength test, while 444 participated in the tests of all 5 muscle groups (Appendix 1). All participants signed an informed consent prior to the examinations.

\section{Clinical assessment}

The presence of chronic medical conditions, with a minimum duration of three months, was ascertained by a physician in a clinical examination. Sixty-two percent of the participants suffered from cardiovascular diseases, such as ischemic heart disease and hypertension. Additionally, musculoskeletal diseases (e.g. osteoarthritis) were present in $39 \%$ of the participants, and neurological (e.g. cerebrovascular ischemia) and sensory (e.g. glaucoma and 
cataract) diseases in $26 \%$ of the participants. Less prevalent disease categories were metabolic diseases (15\%; e.g. diabetes), respiratory diseases (12\%), diseases in the digestive system (10\%), mental diseases (4\%), cancer (9\%), and other non-classified diseases (17\%). Additionally, the physician assessed contraindications to the muscle strength tests.

\section{Physical activity}

A modified version of the multiple-choice question developed and validated by Grimby (1986) and Mattiasson-Nilo et al. (1990) was used to assess the level of physical activity related to leisure time, work and carrying out daily activities. The participants were asked to choose the description that best pictured their level of physical activity over the last year: 1) hardly any activity, mostly sitting; 2) light physical activity, such as light household tasks; 3) moderate physical activity about 3 hours a week: walking longer distances, cycling and domestic work; 4) moderate physical activity at least 4 hours a week or heavier physical activity 1 to 2 hours a week; 5) heavier physical activity or moderate exercise for at least 3 hours a week; and 6) competitive sports. This scale is feasible in older independent populations as it is easy and quick to use and it also rates domestic activities. To avoid small group sizes in the analyses the scale was dichotomized. Participants were considered physically active if they reported moderate physical activity for at least 4 hours a week. The test-retest Pearson correlation coefficients were $\mathrm{r}=0.634$ for men and $\mathrm{r}=0.655$ for women (Sihvonen et al., 1998).

\section{Muscle strength}

Maximal isometric strength of handgrip, elbow flexion and knee extension were measured on the dominant side in a sitting position using an adjustable dynamometer chair, and expressed in Newton (N) (Heikkinen et al., 1984). For the measurement of handgrip strength a dynamometer was fixed to the arm of the chair. Elbow flexion strength was measured with a 
strain-gauge system at the wrist, the elbow supported at an angle of $90^{\circ}$ and the hand in the neutral position (thumb up). Knee extension strength was measured at a knee angle of $60^{\circ}$ from the fully extended leg towards flexion. The ankle was attached to a strain-gauge system. Maximal isometric trunk flexion and trunk extension strength were measured in a standing position according to Viitasalo et al. (1977).

After two to three practise trials, each strength test was performed three times with an intertrial rest period of one minute. The best result was used as the measure of maximal strength in the analyses. The test-retest Pearson correlation coefficients were $r=0.97$ for handgrip, knee extension and trunk flexion strength, and $\mathrm{r}=0.90$ for elbow flexion, and $\mathrm{r}=0.92$ for trunk extension (Rantanen et al., 1997). As muscle strength is highly dependent on gender and age, tertile cut-off points were determined for 75- and 80-year-old men and women separately (Appendix 1). In this way an equal number of men and women were placed in each tertile.

\section{Confounders}

The number of chronic diseases, obtained from the clinical assessment, was used as an indicator of morbidity. Depressive symptoms, smoking and the level of education were assessed by means of a questionnaire. The self-rated Center for Epidemiologic Studies -Depression Scale (CES-D; Radloff, 1977) was used to assess the presence of depressive symptoms (cut-off score 16). The respondents were asked to rate the frequency, ranging from 0 (rarely or none of the time) to 3 (most or all of the time), of 20 listed symptoms over the past week. Additionally, participants were classified as 1) nonsmoker and 2) previous or current smoker and the level of education was described as 1) less than primary school, 2) primary or junior high school, 3) senior high school, or 4) technical school or university. Age and gender were registered and body height and weight were measured at the research laboratory. 


\section{Mortality}

All-cause mortality was followed-up at 10 years from baseline. Death dates were received from the Population Register. Survival time was calculated from baseline to the day the subject died. Subjects not known to have died or lost to the follow-up were given a survival time of 10 years.

\section{Statistical methods}

The gender-specific differences in characteristics of the sedentary and physically active groups were determined by an independent T-test, or cross-tabulation with a Chi-square test. Mortality rates relative to survival time were calculated and expressed as the number of deaths/100 person years.

Cox proportional hazard analyses were first executed separately for both age and gender groups to investigate the association of the level of physical activity and muscle strength, separately and combined, with all-cause mortality. Eventually, the data were combined in a single model, as the direction of the associations was similar and the group sizes were small for meaningful separate analysis. The results were adjusted for age, gender, body height and weight, number of chronic diseases, presence of depressive symptoms, smoking and level of education.

SPSS computer software was used for the analyses. Significance was set at $\mathrm{p}<0.05$. 


\section{Results}

In this study, $38 \%$ of the men and $31 \%$ of the women were considered to be physically active. These men and women reported to engage in moderate physical activity for at least 4 hours a week (28\% and $29 \%$, respectively) or in heavier physical activity and moderate exercise for at least 3 hours a week (10\% and 3\%, respectively). None of the participants took part in competitive sports. The sedentary men and women (in total 62\% and 69\%, respectively) reported hardly any activity (12\% and 6\%, respectively), light physical activity (19\% and $21 \%$, respectively) or moderate physical activity about 3 hours a week ( $33 \%$ and $42 \%$, respectively). The sedentary participants had lower strength in all the muscle groups (Table 1). However, among the women some of the strength differences did not reach statistical significance. Depressive symptoms were more common among the sedentary men (37\%) than active men (20\%, $\mathrm{p}=0.024)$, whereas among both the sedentary and active women the corresponding prevalence was $40 \%$. Twenty-nine percent of the men and $92 \%$ of the women had never smoked.

During the 10-year follow-up period, $56 \%$ of the participants included in the analyses died (men: 63 aged 75 years and 48 aged 80 years; and women: 89 aged 75 years and 111 aged 80 years). After checking that the association of physical activity with mortality was similar in both the gender and age groups, all participants were jointly included in subsequent analyses. In the sedentary participants, the relative risk for mortality (RR) was 1.7 times (95\% confidence interval: 1.3-2.2) that of physically active participants and adjusting for confounders did not change the risk estimate ( $R R=1.7,95 \%$ confidence interval: $1.2-2.2)$. Table 2 presents the crude and adjusted mortality risks according to the level of strength in each muscle group. Crude RR for death was approximately twofold among those in the lowest muscle strength tertile compared 
to the highest, adjusting for confounders did not materially change the estimates. Similar results were obtained for all muscle groups.

To study the combined effects of the muscle strength tertiles and physical activity categories six groups were formed for each muscle group tested. For example, for grip strength, $78 \%$ of those in the lowest tertile were sedentary, while the corresponding figures in the middle and highest tertile were $65 \%$ and $58 \%$, respectively. Figure 1 shows the mortality rates of sedentary and physically active men and women by the strength tertiles in each muscle group. A highly similar mortality rate pattern according to muscle strength and physical activity was observed for men and women even though the rates were generally lower in women. Within each strength tertile, the mortality rate was lower among the physically active than sedentary participants. This difference was particularly evident among those in the lowest strength tertile where the overall mortality rate was higher. Among those in the highest strength tertile with a lower overall mortality rate, physical activity was not equally systematically associated with a lower death rate. The crude and adjusted RR's of mortality according to the level of strength in the groups of sedentary and physically active are shown in Table 2 . For each group of muscles tested, the crude risk for death was 2-3 times higher among sedentary participants in the lowest strength tertile than among physically active participants in the highest strength tertile. The results show that the elevated risk of death associated with lower strength was substantially lower among those who were physically active. However, among those with higher strength, the risks of death were generally lower and physical activity did not show a noticeable association with reduced risk. Adjusting for the confounders did not materially change the results. 


\section{Discussion}

We found that a high level of physical activity may compensate for the increased mortality risk among older people with poorer muscle strength. To our knowledge, this was the first study to determine the combined effects of physical activity and muscle strength on all-cause mortality, an indicator of general health, in older people. However, many longitudinal studies, including this one, using a large variety of measures, have found that poor muscle strength (e.g. Metter et al., 2002; Rantanen et al., 2003) and low physical activity (e.g. Lee \& Skerrett, 2001, for review) are consistently associated with an increased risk for mortality.

Muscle strength is a potential marker for resiliency to poor health in old age. Our observation that mortality risk was lower among the physically active people with poorer strength than among the sedentary suggests that physical activity may partly compensate for less favourable expected health trajectory among those with poorer strength. The associations remained after adjusting for morbidity, body height and weight, depressive symptoms, smoking history and educational background. Age did not affect the associations either, as the age-range was relatively small. The severity of the diseases could not be taken into account and therefore, some residual confounding may have remained in the models. However, we do not believe that disease severity would fully explain the current findings. Taking into account the cause of death could have added information about the pathway leading to death. However, since the independent effects of muscle strength (Rantanen et al., 2003) and physical activity (Kaplan et al., 1996; Bijnen et al., 1998; Greg et al., 2003) on all-cause and cause-specific mortality have been found to be similar, the results of this study would likely not have changed.

Participants were considered physically active if they reported moderate physical activity for at least 4 hours a week, which is approximately the same amount of activity as currently 
recommended for maintaining a healthy life. Leisure walking is the most common activity among older people (DiPietro, 2001, Taylor et al., 2004); however, its intensity may not be high enough for it to be associated with higher muscle strength (American College of Sports Medicine, 1998). The health benefits, including reduced mortality, associated with physical activity were thus more likely to be induced by improvements in cardio-vascular and respiratory function.

Having high muscle strength and being physically active did not seem to have an additional effect in reducing mortality in this study population. However, high levels of both muscle strength and physically activity may be important for other health outcomes or for disability.

The amount of physical activity needed to maintain good health in older age is still unclear, and currently there is no standardized method to assess physical activity in older people. In this study, physical activity was assessed by a short and easy self-report question, which was dichotomized for the analyses. Even this rough indicator of physical activity was predictive of mortality. Potentially larger effects of physical activity on mortality may be found when a more precise measure, differentiating people more adequately, is used. The association between isometric muscle strength and mortality was similar in the five muscle groups. Additionally, $83.1 \%$ of the participants were assigned to the same category for at least 3 muscle groups, while $8.3 \%$ had at least 1 measure missing. These missing values altered the distribution of the data in the respective muscle groups. Handgrip strength may be preferred as indicator of general muscle strength, considering its frequent use in previous studies (Philips, 1986; Fujita et al., 1995; Al Smih et al., 2002; Metter et al., 2002; Rantanen et al., 2003) and its high correlation with muscle strength in other muscle groups (Rantanen et al., 1997). Additionally, handgrip strength is easy and quick to assess. 
The population used in this study was representative of the urban, community-dwelling population of this age in Central Finland. However, we believe that the range of muscle strength was truncated at the lower end, as those unable to attend the laboratory for the examinations and those with contraindications for the muscle strength assessments generally had poorer health status. Of those unable to participate in the muscle strength tests, $68 \%$ were 80 years old, and the mortality rate was 12.8 deaths/100 person years among the men and 8.3 deaths/100 person years among the women. In comparison, men with at least 1 muscle strength measurement had a mortality rate of 6.7 deaths/100 person years and women a mortality rate of 5.2 deaths/100 person years, respectively. The mortality rate and risk estimates found in this study may therefore underestimate the effect of muscle strength.

The mortality rates in were strikingly similar in the different muscle groups, and in men and women. However, considering the small group sizes, especially those of active men, further study is warranted. Larger studies are needed to allow for gender specific analyses and more comprehensive analysis of confounding factors.

\section{Perspectives}

Low level of physical activity (e.g. Lee \& Skerrett, 2001, for review) and muscle strength (Philips, 1986; Fujita et al., 1995; Al Smih et al., 2002; Metter et al., 2002; Rantanen et al., 2003) are acknowledged risk factors for mortality. This study showed that physical activity may counteract the increased mortality risk associated with poor muscle strength. Physical activities, not specifically targeting to increase muscle strength, such as leisure walking, are likely to induce health benefits, reflected in reduced mortality, as the effects of high muscle strength and high physical activity were not additive. Especially, people with low muscle strength are likely to benefit from strategies promoting high levels of physical activity and should therefore be 
targeted for prevention and intervention. However, further study is needed to determine the mechanisms through which physical activity and muscle strength jointly affect mortality and health in older men and women. 


\section{Acknowledgements}

The Evergreen study has been supported by the Academy of Finland, the Ministry of Education and the Ministry of Social Affairs and Health, the Social Insurance Institution, the Association of the Finnish Lions Clubs and the City of Jyväskylä. 


\section{References}

Al Smih S, Markides KS, Ray L, Ostir GV, Goodwin JS. Handgrip strength and mortality in older Mexican Americans. J Am Geriatr Soc 2002: 50: 1250-1256.

American College of Sports Medicine Position Stand. Exercise and physical activity for older adults. Med Sci Sports Exerc 1998: 30: 992-1008.

Bean JF, Vora A, Frontera WR. Benefits of exercise for community-dwelling older adults. Arch Phys Med Rehabil 2004: 85(Suppl 3): S31-42.

Bijnen FCH, Caspersen CJ, Feskens EJM, SarisWHM, Mosterd WL, Kromhout D. Physical activity and 10-year mortality from cardiovascular diseases and all causes. Arch Intern Med 1998: 158: 1499-1505.

DiPietro L. Physical activity in aging: changes in patterns and their relationship to health and function. J Gerontol 2001:56A(special issue II):13-22.

Fujita Y, Nakamura Y, Hiraoka J, et al. Physical-strength tests and mortality among visitors to health-promotion centers in Japan. J Clin Epidemiol 1995: 48: 1349-1359.

Gregg EW, Cauley JA, Stone K et al. Relationship of changes in physical activity and mortality among older women. JAMA 2003: 289: 2379-2386.

Grimby G. Physical activity and muscle training in the elderly. Acta Med Scand 1986:

711(suppl): 233-237.

Heikkinen E. Background, design and methods of the project. Scand J Soc Med 1997: 53(Suppl): $1-18$.

Heikkinen E, Arajärvi R-L, Era P, et al. Functional capacity of men born in 1906-10, 1926-30, 1946-50. A basic report. Scand J Soc Med 1984: 33(suppl): 1-93. 
Kaplan GA, Strawbridge WJ, Cohen RD, Hungerford LR. Natural history of leisure-time physical activity and its correlates: Associations with mortality form all causes and cardiovascular disease over 28 years. Am J Epidemiol 1996: 144: 793-797.

Kushi LH, Fee RM, Folmsom AR, Mink PJ, Anderson KE, Sellers TA. Physical activity and mortality in postmenopausal women. JAMA 1997: 277: 1287-1292.

Lee IM, Skerrett PJ. Physical activity and all-cause mortality: what is the dose-response relation? Med Sci Sports Exerc 2001: 33(suppl): S459-471.

Mattiasson-Nilo I, Sonn U, Johannesson K, Gosman-Hedstrom G, Persson GB, Grimby G. Domestic activities and walking in the elderly: evaluation from a 30-hour heart rate recording. Aging 1990: 2:191-198.

Metter EJ, Talbot LA, SChrager M, Conwit R. Skeletal muscle strength as a predictor of allcause mortality in healthy men. J Gerontol A Biol Sci Med Sci 2002: 57: B359-365.

Philips P. Grip strength, mental performance and nutritional status as indicators of mortality risk among female geriatric patients. Age Aging 198: 15: 53-56.

Radloff LS. The CES-D scale: a self-report depression scale for research in the general population. Appl Psychol Meas 1977: 1: 385-401.

Rantanen T, Era P, Heikkinen E. Maximal Isometric Strength and mobility among 75-year-old men and women. Age Ageing 1994: 23: 132-137.

Rantanen T, Era P, Heikkinen E. Physical activity and the change in maximal isometric strength in men and women from the age of 75 to 80 years. J Am Geriatr Soc 1997: 45: 1439-1445. Rantanen T, Heikkinen E. The role of habitual physical activity in preserving muscle strength from age 80 to 85 years. J Aging Phys Act 1998: 6: 121-132. 
Rantanen T, Volpato S, Ferrucci L, Heikkinen E, Fried LP, Guralnik JM. Handgrip strength, cause-specific and total mortality in older disabled women: exploring the mechanism. J Am Ger Soc 2003: 51: 636-641.

Sihvonen S, Rantanen T, Heikkinen E. Physical activity and survival in elderly people: A fiveyear follow-up study. J Aging Phys Act 1998: 6: 133-140.

Taylor AH, Cable NT, Faulkner G, Hillsdon M, Narici M, van der Bij AK. Physical activity and older adults: a review of health benefits and the effectiveness of interventions. J Sports Sci 2004: 22: 703-725.

Tiainen K, Sipilä S, Alen M et al. Heritability of maximal isometric strength in older female twins. J Appl Physiol. 2004 Jan: 96: 173-180.

Viitasalo J, Saukkonen S, Komi P. Physical health and performance capacity, and physical activity habits in conscripts in the beginning of service at Air Force Communication School, Research reports from the department of Biology of Physical Activity, University of Jyväskylä, 1977. 
Table 1: Means and standard deviations (SD) of the baseline characteristics of sedentary and physically active 75- and 80-year-old men and women with at least 1 muscle strength measure.

\begin{tabular}{|c|c|c|c|c|c|c|c|c|c|c|}
\hline & \multicolumn{5}{|c|}{ Men } & \multicolumn{5}{|c|}{ Women } \\
\hline & \multicolumn{2}{|c|}{ Sedentary } & \multicolumn{2}{|r|}{ Active } & \multirow{2}{*}{$\begin{array}{r}\text { t-test } \\
p\end{array}$} & \multicolumn{2}{|c|}{ Sedentary } & \multicolumn{2}{|r|}{ Active } & \multirow{2}{*}{${ }^{\mathrm{t} \text {-test }}$} \\
\hline & $\mathrm{n}$ & Mean \pm SD & $\mathrm{n}$ & Mean \pm SD & & $\mathrm{n}$ & Mean \pm SD & $\mathrm{n}$ & Mean \pm SD & \\
\hline Body Height (cm) & 98 & $169.2 \pm 6.6$ & 59 & $169.4 \pm 5.8$ & 0.826 & 221 & $155.8 \pm 5.6$ & 101 & $155.4 \pm 5.4$ & 0.563 \\
\hline Body Weight (kg) & 98 & $75.0 \pm 12.1$ & 59 & $73.4 \pm 9.7$ & 0.387 & 221 & $67.5 \pm 11.9$ & 101 & $63.8 \pm 8.8$ & 0.007 \\
\hline Chronic Diseases (n) & 98 & $2.6 \pm 1.4$ & 58 & $1.8 \pm 1.3$ & 0.002 & 222 & $2.7 \pm 1.7$ & 101 & $2.4 \pm 1.6$ & 0.109 \\
\hline Handgrip Strength (N) & 97 & $343.3 \pm 83.4$ & 59 & $396.0 \pm 86.3$ & $<0.001$ & 221 & $208 \pm 57.6$ & 101 & $227.5 \pm 63.4$ & 0.007 \\
\hline Elbow Flexion Strength (N) & 97 & $231.4 \pm 56.8$ & 59 & $271.3 \pm 42.6$ & $<0.001$ & 222 & $148.2 \pm 40.5$ & 101 & $156.4 \pm 43.2$ & 0.100 \\
\hline Knee Extension Strength (N) & 96 & $318.2 \pm 104.1$ & 59 & $378.7 \pm 81.4$ & $<0.001$ & 220 & $218.1 \pm 71.2$ & 101 & $232.7 \pm 80.9$ & 0.104 \\
\hline Trunk Extension Strength(N) & 87 & $478.5 \pm 214.4$ & 58 & $657.1 \pm 176.9$ & $<0.001$ & 207 & $293.4 \pm 133.8$ & 97 & $326.1 \pm 140.9$ & 0.051 \\
\hline Trunk Flexion Strength (N) & 88 & $459.4 \pm 176.7$ & 57 & $544.2 \pm 133.0$ & 0.002 & 204 & $270.2 \pm 96.0$ & 95 & $294.6 \pm 112.6$ & 0.053 \\
\hline
\end{tabular}

* physically active: moderate physical activity at least 4 hours a week: sedentary: about 3 hours of moderate physical activity a week

or less 
Table 2: Relative risks (RR) for mortality and 95\% confidence intervals (95\%CI)* according to muscle strength tertiles for each muscle group (all) and for groups based on physical activity and muscle strength.

\begin{tabular}{|c|c|c|c|c|c|c|c|c|c|c|c|c|c|}
\hline & \multirow[b]{3}{*}{ Tertile } & \multicolumn{6}{|c|}{ Crude } & \multicolumn{6}{|c|}{ Adjusted $^{\S}$} \\
\hline & & \multirow{2}{*}{\multicolumn{2}{|c|}{$\begin{array}{l}\text { All } \\
95 \% \mathrm{CI}\end{array}$}} & \multicolumn{2}{|c|}{ Sedentary \# } & \multicolumn{2}{|c|}{ Active $^{+}$} & \multicolumn{2}{|r|}{ All } & \multicolumn{2}{|c|}{ Sedentary } & \multicolumn{2}{|c|}{ Active } \\
\hline & & & & $\mathrm{RR}$ & $95 \% \mathrm{CI}$ & $\mathrm{RR}$ & $95 \% \mathrm{CI}$ & $\mathrm{RR}$ & $95 \% \mathrm{CI}$ & $\mathrm{RR}$ & $95 \% \mathrm{CI}$ & RR & $95 \% \mathrm{CI}$ \\
\hline \multirow[t]{3}{*}{ Handgrip } & Lowest & 1.8 & $1.3-2.5$ & 2.2 & $1.5-3.4$ & 1.2 & $0.7-2.2$ & 1.7 & $1.2-2.5$ & 2.0 & $1.2-3.3$ & 1.2 & $0.6-2.4$ \\
\hline & Medium & 1.3 & $0.9-1.7$ & 1.7 & $1.1-2.6$ & 0.9 & $0.5-1.6$ & 1.4 & $1.0-2.0$ & 1.8 & $1.1-2.9$ & 1.0 & $0.5-1.8$ \\
\hline & Highest & 1.0 & & 1.1 & $0.7-1.8$ & 1.0 & & 1.0 & & 1.2 & $0.7-2.0$ & 1.0 & \\
\hline Elbow & Lowest & 2.0 & $1.5-2.7$ & 2.7 & $1.7-4.2$ & 1.2 & $0.6-2.3$ & 1.9 & $1.3-2.7$ & 2.6 & $1.6-4.3$ & 1.4 & $0.7-2.9$ \\
\hline \multirow[t]{2}{*}{ Flexion } & Medium & 1.3 & $1.0-1.8$ & 1.7 & $1.1-2.7$ & 1.2 & $0.7-2.1$ & 1.3 & $0.9-1.8$ & 1.8 & $1.1-2.9$ & 1.2 & $0.7-2.2$ \\
\hline & Highest & 1.0 & & 1.2 & $0.8-2.0$ & 1.0 & & 1.0 & & 1.4 & $0.8-2.4$ & 1.0 & \\
\hline Knee & Lowest & 2.2 & $1.6-3.0$ & 3.3 & $2.0-5.3$ & 1.9 & $1.1-3.6$ & 2.0 & $1.4-2.9$ & 3.0 & $1.8-5.1$ & 2.1 & $1.1-4.2$ \\
\hline \multirow[t]{2}{*}{ Extension } & Medium & 1.8 & $1.3-2.5$ & 2.6 & $1.6-4.2$ & 1.9 & $1.0-3.3$ & 1.7 & $1.2-2.5$ & 2.6 & $1.5-4.4$ & 1.9 & $1.0-3.6$ \\
\hline & Highest & 1.0 & & 1.5 & $0.9-2.6$ & 1.0 & & 1.0 & & 1.7 & $1.0-3.0$ & 1.0 & \\
\hline Trunk & Lowest & 2.3 & $1.6-3.2$ & 2.9 & $1.8-4.6$ & 2.2 & $1.2-4.1$ & 1.9 & $1.3-2.8$ & 2.3 & $1.4-3.9$ & 1.8 & $0.9-3.8$ \\
\hline Extension & Medium & 1.5 & $1.0-2.1$ & 2.1 & $1.3-3.3$ & 1.3 & $0.7-2.3$ & 1.4 & $1.0-2.1$ & 2.1 & $1.2-3.5$ & 1.1 & $0.6-2.0$ \\
\hline
\end{tabular}




\begin{tabular}{llllllllllllll}
\hline \multirow{2}{*}{ Trunk } & Highest & 1.0 & & 1.4 & $0.8-2.4$ & 1.0 & & 1.0 & & 1.3 & $0.7-2.3$ & 1.0 & \\
\multirow{2}{*}{ Flexion } & Lowest & 1.9 & $1.3-2.6$ & 2.8 & $1.7-4.4$ & 1.5 & $0.8-2.8$ & 1.6 & $1.1-2.4$ & 2.3 & $1.4-3.9$ & 1.2 & $0.6-2.4$ \\
& Medium & 1.3 & $0.9-1.8$ & 1.8 & $1.1-3.0$ & 1.3 & $0.7-2.4$ & 1.1 & $0.8-1.7$ & 1.6 & $0.9-2.7$ & 1.0 & $0.5-2.0$ \\
& Highest & 1.0 & & 1.5 & $0.9-2.5$ & 1.0 & & 1.0 & & 1.4 & 1.0 & \\
\hline
\end{tabular}

* obtained from Cox proportional hazard analysis

$\S$ adjusted for age, gender, body height and weight, number of diseases, presence of depressive symptoms, smoking and education level

\# sedentary: about 3 hours of moderate physical activity a week or less

${ }^{+}$active: moderate physical activity at least 4 hours a week 
Figure 1: Mortality rate (number of deaths / 100 person years over follow-up) in sedentary and physically active (moderate physical activity for at least 4 hours a week) men and women according to strength tertiles for each muscle group. The number of deaths

relative to the group size is noted at the end of each bar. 
Women

\section{Men}

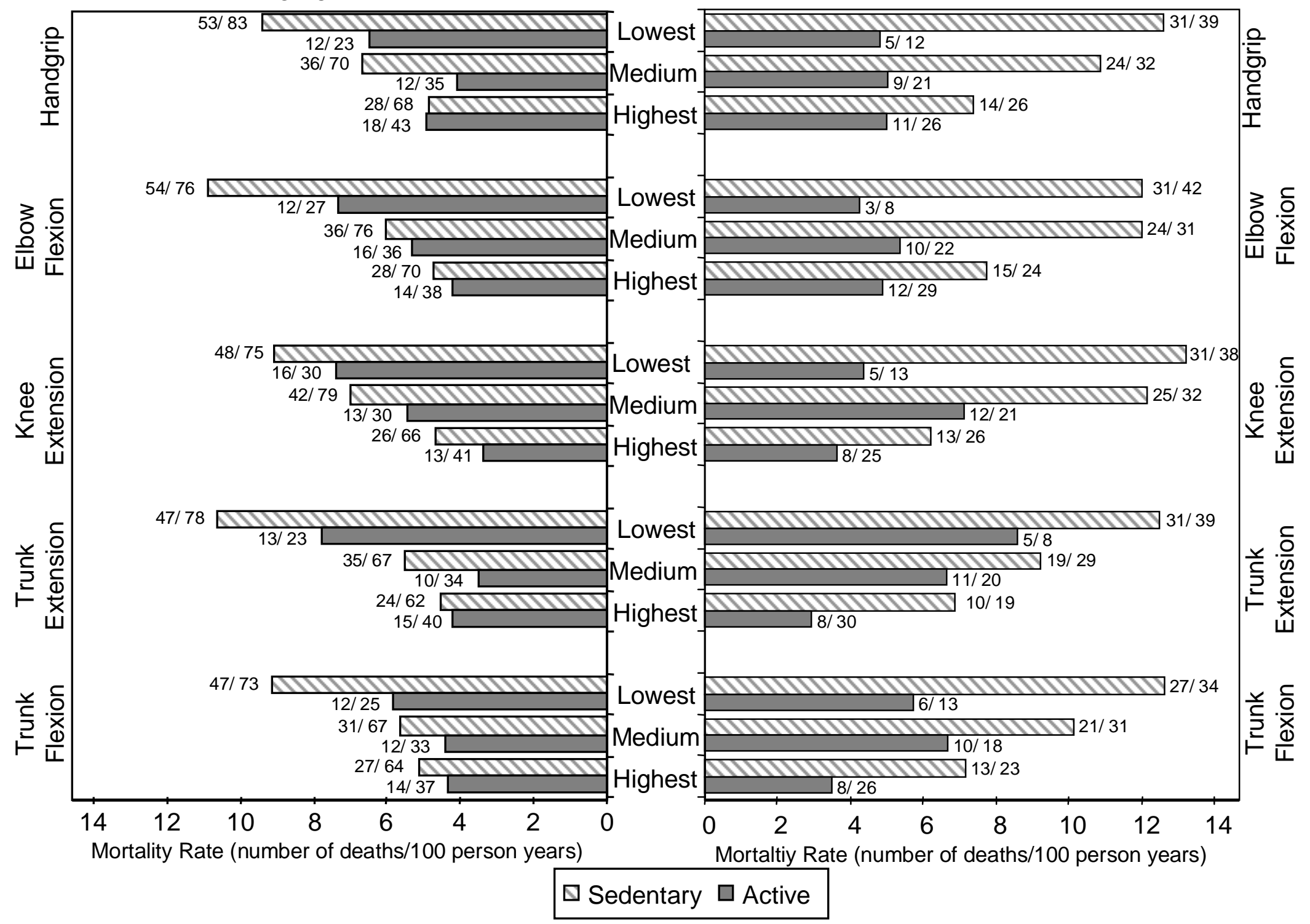


Appendix 1. Age- and gender-specific cut-off points for strength, expressed in Newton, in each muscle group.

Men

Women

Tertile 75-year-old $\quad 80$-years-old $\quad 75$-year-old $\quad 80$-years-old

Handgrip $(\mathrm{n}=478)$

Lowest

$<328.0$

$<316.3$

$<205.0$

$<177.3$

Medium

328.0- 404.0

316.3- 371.3

205.0- 253.0

177.3- 219.0

Highest

$\geq 404.0$

$\geq 371.3$

$\geq 253.0$

$\geq 219.0$

Elbow Flexion $(n=478)$

Lowest

$<231.0$

$<206.0$

$<144.0$

$<118.3$

Medium

231.0- 267.0

206.0- 266.0

144.0- 178.0

118.3- 156.7

Highest

$\geq 276.0$

$\geq 266.0$

$\geq 178.0$

$\geq 156.7$

Knee Extension $(n=476)$

Lowest

Medium

Highest

Trunk Extension $(\mathrm{n}=449)$

Lowest

Medium

Highest

Trunk Flexion $(\mathrm{n}=444)$

Lowest

$<455.0$

$455.0-590.0$

$\geq 681.0$

Medium
$<375.0$

$<285.0$

$<183.3$

504.0- $681.0 \quad 375.0-559.0 \quad 285.0-409.7 \quad 183.3-278.3$

$\geq 559.0$

$\geq 409.7$

$\geq 278.3$ 
